\title{
Temperatura base para emissão de nós e plastocrono de plantas de melancia $^{1}$
}

\author{
Base temperature for node appearance and plastochron of watermelon plant
}

\author{
Dionéia Daiane Pitol Lucas², Nereu Augusto Streck ${ }^{3}$, Mateus Possebon Bortoluzzi ${ }^{4}$, Roberto Trentin ${ }^{5}$ e Ivan \\ Carlos Maldaner ${ }^{6 *}$
}

\begin{abstract}
Resumo - O efeito da temperatura do ar sobre o desenvolvimento das plantas pode ser representado usando-se o método da soma térmica. Para o cálculo da soma térmica é necessário conhecer-se a temperatura base, abaixo da qual o desenvolvimento não acontece ou acontece a uma taxa desprezível. O objetivo deste trabalho foi estimar a temperatura base para emissão de nós, bem como comparar as datas de cultivo para a variável plastocrono para a cultura da melancia. Três experimentos em campo foram conduzidos em Santa Maria, RS, com oito datas de semeadura durante os anos agrícolas 2006-2007 (05/09/2006 e 21/09/2006), 2008/2009 (20/09/2008, 06/10/2008 e 20/10/2008) e 2009/2010 (20/09/2009, 21/10/2009 e 30 /11/2009). Usou-se o cultivar "Crimson Sweet" aleatorizado em blocos ao acaso com quatro repetições por época. O número de nós acumulados na haste principal (NN) da melancieira foi observado em três plantas por parcela, três vezes por semana. O plastocrono foi estimado pelo inverso do coeficiente angular da regressão entre $\mathrm{NN}$ e soma térmica acumulada. A temperatura base estimada usando a metodologia do menor valor de Quadrado Médio do Erro (QME) foi de 7,0 ${ }^{\circ} \mathrm{C}$. O plastocrono em melancieira não diferiu entre as datas de plantio nos três anos agrícolas, sendo em média $23,4^{\circ} \mathrm{C}$ dia nó ${ }^{-1}$. A implicação para a modelagem é que um único valor de plastocrono pode ser usado para estimar a emissão de nós da cultivar "Crimson Sweet", independente da data de semeadura.
\end{abstract}

Palavras-chave - Citrullus lanatus. Desenvolvimento vegetativo. Soma térmica.

\begin{abstract}
The air temperature effect on plant development can be represented using the thermal time approach. In order to calculate the thermal time, the base temperature, below when development stops or takes place at very low rates, is needed. This work was carried out to estimate the base temperature for node appearance and compared dates for cultivation for variable plastochron in watermelon crop. Three field experiments were conducted in Santa Maria, RS, Brazil, with eight sowing dates during the 2006-2007 growing season (05/09/2006 and 21/09/2006), 2008/2009 (20/09/2008, 06/10/2008, and 20/10/2008) and 2009/2010 (20/09/2009, 21/10/2009, and 30/11/2009). The cultivar "Crimpson Sweet" was used in a randomized block design with four replications per sowing dates. The accumulated number of nodes on the main stem (NN) of watermelon was observed in three plants, three times a week. Plastochron was estimated by the inverse of the slope in the linear regression of $\mathrm{NN}$ against accumulated thermal time. The temperature base estimated using the least Mean Square Error (MSE) approach was $7.0^{\circ} \mathrm{C}$. The plastochron in watermelon was not different among sowing dates during the three growing seasons, with an average of $23.4^{\circ} \mathrm{C}$ day node ${ }^{-1}$. From a modeling perspective, the implications of these results are that a single plastochron value can be used to simulate node appearance of the "Crimpson Sweet" cultivar, regardless of the sowing date.
\end{abstract}

Key words - Citrullus lanatus. Vegetative development. Thermal time.

\footnotetext{
*Autor para correspondência

${ }^{1}$ Recebido para publicação em 24/08/2010; aprovado em 10/08/2011

Pesquisa financiada pela FAPERGS, CAPES e CNPq

${ }^{2}$ Programa de Pós-Graduação em Agronomia, Universidade Federal de Santa Maria, Santa Maria-RS, Brasil, dio.pitol@gmail.com

${ }^{3}$ Departamento de Fitotecnia, Universidade Federal de Santa Maria, Santa Maria-RS, Brasil, nstreck@yahoo.com.br

${ }^{4}$ Graduando do Curso de Agronomia, Universidade Federal de Santa Maria, Santa Maria-RS, Brasil, mateusbortoluzzi@ hotmail.com

${ }_{5}^{5}$ Programa de Pós-Graduação em Engenharia Agrícola, Universidade Federal de Santa Maria, Santa Maria-RS, Brasil, robertotrentin@yahoo.com.br

${ }^{6}$ Instituto Federal Farroupilha-Campus São Vicente do Sul, São Vicente do Sul-RS, Brasil, 97.420-000, ivan_maldaner@yahoo.com.br
} 


\section{Introdução}

A melancieira (Citrullus lanatus Schrad) tem origem na África tropical e pertence à família das cucurbitáceas, sendo o cultivo anual, com ciclo variando entre 80 e 110 dias (CARVALHO, 1999). A produção de melancia no Brasil ocupa o quarto lugar dentre as olerícolas, sendo esta de 1,995 mil t em 89 mil ha (EMBRAPA, 2008). Esta produção é considerada baixa, estando associada a plantios pouco tecnificados e também à falta de irrigação em algumas regiões (LEÃO, 2008), além da falta de aplicação de conhecimentos simples, como o de que o aumento de espaçamento entre plantas aumenta a produção consideravelmente (BASTOS et al., 2008). O Rio Grande do Sul é o estado com maior produção, respondendo por $28,5 \%$ da produção brasileira em 2008 (IBGE, 2008). O cultivar Crimson Sweet é o mais cultivado em Santa Maria e demais regiões do estado (TRENTIN et al., 2008), apresenta adaptação às variações de cultivo e atende às exigências do consumidor brasileiro (LEONEL et al., 2000).

A caracterização do desenvolvimento vegetal auxilia na elaboração e aplicação de estratégias de manejo nas culturas agrícolas, referindo-se a eventos que envolvem desde diferenciação celular até senescência da planta (HODGES, 1991). A temperatura do ar é o principal elemento meteorológico que afeta o desenvolvimento das culturas agrícolas (STRECK, 2002; GRAMIG; STOLTENBERG, 2007).

O conceito da soma térmica ou dos grausdia é muito utilizado para a quantificação do efeito da temperatura do ar sobre o desenvolvimento das plantas (GILMORE; ROGERS, 1958; ARNOLD, 1960; JEFFERIES; MACKERRON, 1987), sendo uma melhor medida de tempo biológico que dias do calendário civil ou dias após a semeadura (GILMORE; ROGERS, 1958). A clássica equação de cálculo da soma térmica considera a acumulação dos valores de temperatura média diária do ar acima de uma temperatura base (Tb) (McMASTER; WILHELM, 1997). Portanto, para o cálculo da soma térmica é necessário conhecer-se a $\mathrm{Tb}$ que rege $\mathrm{o}$ desenvolvimento de determinada espécie.

Segundo Trentin et al. (2008), a implantação da cultura e as práticas de manejo podem ser melhoradas com auxílio de modelos matemáticos, como na prática da irrigação para a qual se utilizam as informações referentes ao coeficiente de cultura $(\mathrm{Kc})$, variável segundo o estágio de desenvolvimento da cultura considerado (CARVALHO et al., 2007). Dessa forma, a soma térmica é um parâmetro que pode ser usado para a redução de riscos climáticos, uma vez que o conhecimento das exigências térmicas de uma cultura contribui para a previsão da duração do ciclo da planta (BARBANO et al., 2001). Além disso, quando cultivada na época recomendada, a soma térmica funciona bem como preditor dos diferentes estágios do ciclo de desenvolvimento (STRECK et al., 2005).

A emissão de nós pode ser estimada a partir do conhecimento do tempo necessário para o aparecimento de dois nós sucessivos na planta, o que é uma excelente medida de desenvolvimento vegetal (STRECK et al., 2003). Em dicotiledôneas, o intervalo de tempo entre o aparecimento de nós sucessivos na haste é denominado de plastocrono (BAKER; REDDY, 2001).

$\mathrm{Na}$ literatura a soma térmica das fases foi estimada utilizando-se valor de Tb para a cultura do melão (TRENTIN et al., 2008). Partindo-se da hipótese de que há variações de Tb para diferentes espécies (MARTINS et al., 2007), o objetivo deste trabalho foi estimar a temperatura base para emissão de nós, bem como comparar as datas de cultivo para a variável plastocrono para a cultura da melancieira.

\section{Material e métodos}

Foram utilizados neste trabalho dados de experimentos com a cultura da melancia em diferentes épocas de semeadura, conduzidos em área experimental localizada em Santa Maria, RS (latitude: 29 43' S, longitude: $53^{\circ} 43^{\prime} \mathrm{W}$ e altitude: $95 \mathrm{~m}$ ), durante os anos agrícolas de 2006/2007, 2008/2009 e 2009/2010. Foi utilizada a cultivar de melancia Crimson Sweet, em duas datas de semeadura no primeiro ano (05/09/2006 e 21/09/2006), três datas de semeadura no segundo ano (20/09/2008, 06/10/2008 e 20/10/2008) e três datas de semeadura no terceiro ano (20/09/2009, 21/10/2009 e 30/11/2009).

Oclimadaregiãoé dotipoCfa, conforme aclassificação de Köppen, isto é, subtropical úmido com verões quentes e sem estação seca definida. A média de temperatura do ar para o local, no mês de junho e julho, é de $13,8^{\circ} \mathrm{C}$, enquanto que para o mês de janeiro é de $24,7{ }^{\circ} \mathrm{C}$ e a precipitação normal anual é de 1712,4 mm, distribuída de forma uniforme nas quatro estações do ano (HELDWEIN et al., 2009). O solo representativo do local é um Argissolo Vermelho Distrófico arênico (EMBRAPA, 2006).

A semeadura no cultivo de 2006/2007 foi realizada diretamente no solo, em covas no espaçamento de $1 \mathrm{~m}$ entre fileiras e 1,25 m entre plantas. Foram colocadas três sementes por cova, sendo feito o desbaste quando surgia, nas plântulas, a primeira folha verdadeira, deixando-se somente uma plântula por cova. Já nos anos de 2008/2009 e 2009/2010, a semeadura foi realizada em bandejas preenchidas com substrato Plantmax, mantidas em estufa plástica até o transplante realizado quando as plantas apresentavam duas folhas definitivas, em 22/10/2008, 04/11/2008 e 09/11/2008, no segundo ano, e 29/10/2009, $11 / 11 / 2009$ e 30/12/2009, no terceiro ano, para a primeira, 
segunda e terceira épocas de semeadura, respectivamente, no espaçamento de $1,00 \mathrm{~m}$ entre fileiras e 1,25 m entre plantas. O delineamento experimental utilizado foi $\mathrm{o}$ aleatorizado em blocos, com quatro repetições por época.

A irrigação foi feita pelo método de gotejamento por meio de mangueiras plásticas, visando a manter o solo, empiricamente, próximo à capacidade de campo do solo. As plantas foram mantidas sem competição com plantas daninhas por meio de capinas manuais, além de terem sido realizados os tratos fitossanitários recomendados para a cultura.

$\mathrm{O}$ número de nós (NN) acumulados na haste principal da melancieira foi observado em três plantas por parcela, totalizando 12 plantas por data de semeadura, na frequência de três vezes por semana. Foi considerado visível o nó quando a folha associada a ele apresentava limbo foliar desenrolado e com no mínimo dois centímetros $(2 \mathrm{~cm})$ de largura.

A soma térmica diária ( $\mathrm{STd},{ }^{\circ} \mathrm{C}$ dia) foi calculada de acordo com Arnold (1960):

$\mathrm{STd}=(\mathrm{Tmed}-\mathrm{Tb}) .1 \mathrm{dia}$

em que Tmed é a temperatura média do ar, calculada pela média aritmética entre as temperaturas mínima e máxima diárias do ar, e Tb é a temperatura base para emissão de nós na melancia. A soma térmica acumulada (STa, ${ }^{\circ} \mathrm{C}$ dia), foi calculada acumulando-se os valores de STd desde a emergência das plantas.

A partir dos dados de número de nós, foi realizada a determinação da temperatura base, segundo metodologia que utiliza o menor quadrado médio do erro (QME) da regressão linear entre o NN e a STa (SINCLAIR et al., 2004; MARTINS et al., 2007; PAULA; STRECK, 2008). Seguindo a metodologia utilizada pelos autores citados acima, para o cálculo da STd, foram utilizados os valores de $\mathrm{Tb}$ de $0{ }^{\circ} \mathrm{C}$ até $20^{\circ} \mathrm{C}$ com intervalo de $0,5^{\circ} \mathrm{C}$. Para cada planta, foram estimadas equações de regressão linear simples entre os valores de NN por época em função da STa calculada com as diferentes Tbs. Desta forma, o valor de Tb foi o que apresentou o menor QME nas equações de regressão linear simples.

Com a Tb estimada para a melancia, procedeu-se a estimativa do plastocrono, utilizando-se a regressão linear simples entre NN e STa para cada unidade experimental (parcela com três plantas). O plastocrono $\left({ }^{\circ} \mathrm{C}\right.$ dia nó $\left.{ }^{-1}\right)$ foi considerado como sendo o inverso do coeficiente angular da regressão linear entre NN e STa (BAKER; REDDY, 2001; STRECK et al., 2005). Os dados do plastocrono foram submetidos à análise da variância e as médias das épocas foram comparadas pelo teste Tukey a $5 \%$ de significância.

\section{Resultados e discussão}

As equações de regressão entre NN e STa para melanciativeram valores do coeficiente de determinação $\left(\mathrm{R}^{2}\right)$ acima de 0,92 indicando que a relação de proporcionalidade entre as variáveis analisadas é alta e confirmando que a temperatura do ar é o principal elemento meteorológico que afeta a emissão de nós para a espécie estudada.

Na Figura 1, a título de ilustração, é apresentada a variação do QME das várias equações de regressão para os diferentes valores de temperatura base assumidos no cálculo da soma térmica para uma planta de melancia com semeadura em 05/09/2006. A Tb estimada foi de $9,5^{\circ} \mathrm{C}$, representada pelo marcador de círculo sólido. Para as demais plantas de melancia, houve variação na Tb estimada de zero a $9,5^{\circ} \mathrm{C}$ para a primeira data de semeadura e zero a $16^{\circ} \mathrm{C}$ para a segunda data de semeadura do primeiro ano agrícola de cultivo. Para o segundo ano de experimento, as Tbs variaram, para as diferentes plantas, entre zero e $13,1^{\circ} \mathrm{C}, 2$ e $15,8^{\circ} \mathrm{C}$ e zero a $17,3^{\circ} \mathrm{C}$ para a primeira, segunda e terceira datas de semeadura, respectivamente. Para o terceiro ano, as Tbs variaram entre zero e $20^{\circ} \mathrm{C}$ e zero e $18^{\circ} \mathrm{C}$ para a primeira e segunda datas de semeadura e $20^{\circ} \mathrm{C}$ para todas as plantas na terceira data de semeadura. Apesar da variação grande de Tbs em algumas datas de semeadura, notou-se que a variação do QME entre as Tbs de 5 a $10^{\circ} \mathrm{C}$ foi pequeno na maioria das plantas. Esta foi uma motivação para optar-se fazer a média dos valores de $\mathrm{Tb}$ com menor QME, resultando numa $\mathrm{Tb}$ de

Figura 1 - Quadrado médio do erro (QME) da regressão linear entre o número de nós acumulados na haste principal (NN) e a soma térmica acumulada (STa) para uma planta de melancieira, cultivar "Crimson Sweet", na data de semeadura de 05/09/2006, utilizando-se várias temperaturas base. Santa Maria, RS. O círculo sólido representa o menor QME e a temperatura base de aparecimento de nós

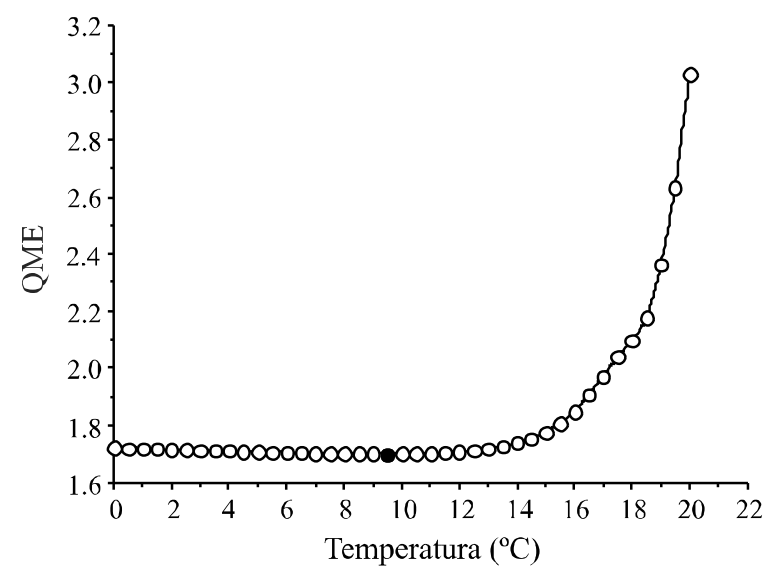


$7,0^{\circ} \mathrm{C}$, valor que foi usado no cálculo da soma térmica para estimativa do plastocrono.

A regressão entre $\mathrm{NN}$ e $\mathrm{STa}$ com $\mathrm{Tb}=7,0{ }^{\circ} \mathrm{C}$ teve valor de $\mathrm{R}^{2}$ elevado (acima de 0,92) para todas as plantas. A título de ilustração é mostrado na Figura 2 esta regressão para uma planta na data de transplante de 06/10/2008. O alto grau de associação entre o número de nós emitidos e a soma térmica $(0,99)$, também verificado para outras espécies vegetais dicotiledôneas como o melão (STRECK et al., 2005), a soja (STRECK, 2008), corriola (PAULA; STRECK, 2008), indica que a $\mathrm{Tb}$ de $7{ }^{\circ} \mathrm{C}$ é apropriada no calculo da STd e que a estimativa do plastocrono pelo método de regressão linear entre NN e STa é apropriado também para a melancia (SINCLAIR et al., 2005; STRECK et al., 2005).

Aexposição a diferentes condições meteorológicas é fundamental nos estudos de avaliação de parâmetros de crescimento e desenvolvimento vegetal e que usam a soma térmica como medida de tempo biológico em plantas (STRECK et al., 2005). Em condições de campo para um mesmo local, consegue-se expor as plantas a diferentes condições ambientais através de semeaduras

Figura 2 - Regressão linear entre o número de nós (NN) e a STa usada na estimativa do plastocrono da melancieira cultivar "Crimson Sweet" com Tb de 7,0 ${ }^{\circ} \mathrm{C}$. Os dados são de uma planta na data de semeadura 06/10/2008. Santa Maria, RS

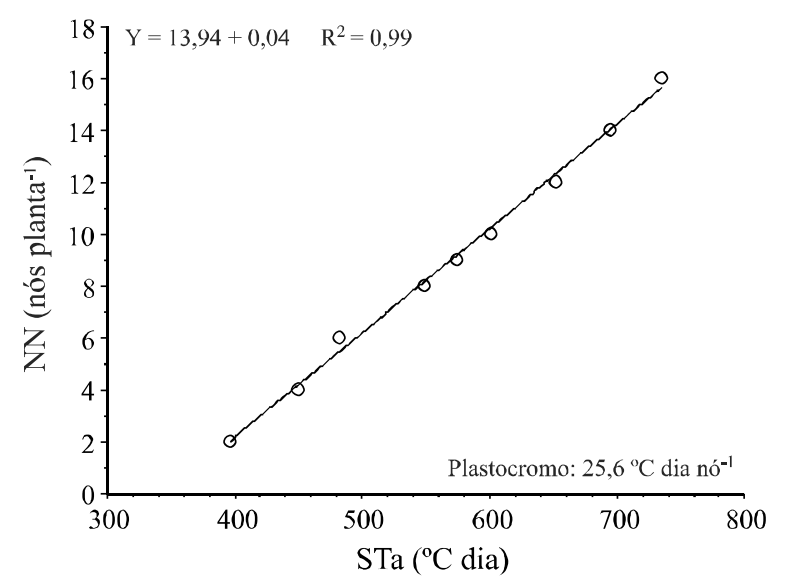

ou plantios em diferentes datas no decorrer do mesmo ano agrícola e através de semeaduras em vários anos. Dessa forma, os valores de plastocrono para as diferentes datas de semeadura nos três anos agrícolas, calculados com $\mathrm{Tb}$ de $7,0^{\circ} \mathrm{C}$ foram submetidos à análise de variância (TAB. 1), não havendo diferença significativa entre as datas avaliadas (TAB. 2).

A média geral de plastocrono nas diferentes datas é $23,3{ }^{\circ} \mathrm{C}$ dia nó ${ }^{-1}$ e não houve diferença entre datas de semeadura (TAB. 1 e 2). A implicação desses resultados é, por exemplo, em estudos de modelagem que usam o conceito de soma térmica e do plastocrono para simular o desenvolvimento vegetativo das culturas agrícolas (STRECK etal., 2005; MARTINS et al., 2007). Como neste caso o plastocrono da melancieira não diferiu entre datas de semeadura nos diferentes anos agrícolas, pode-se usar um único valor de plastocrono para estimar a emissão de nós da cultivar "Crimpson Sweet", independente da data de semeadura, o que é uma vantagem considerável, pois torna o modelo mais geral e, assim, de uso mais amplo.

Tabela 2 - Comparação de médias para plastocrono $\left({ }^{\circ} \mathrm{C}\right.$ dia nó $^{-1}$ ) de melancieira, cultivar "Crimson Sweet", em diferentes datas de semeadura. Santa Maria, 2006/2007; 2008/2009 e $2009 / 2010$

\begin{tabular}{lc}
\hline Data de semeadura & Plastocrono $\left({ }^{\circ} \mathrm{C}\right.$ dia nó $\left.{ }^{-1}\right)$ \\
\hline $05 / 09 / 2006$ & $24,7 \mathrm{a}^{*}$ \\
$21 / 09 / 2006$ & $25,5 \mathrm{a}$ \\
$20 / 09 / 2008$ & $22,2 \mathrm{a}$ \\
$06 / 10 / 2008$ & $22,2 \mathrm{a}$ \\
$20 / 10 / 2008$ & $24,0 \mathrm{a}$ \\
$20 / 09 / 2009$ & $23,2 \mathrm{a}$ \\
$21 / 10 / 2009$ & $22,0 \mathrm{a}$ \\
$30 / 11 / 2009$ & $22,2 \mathrm{a}$ \\
\hline \multirow{2}{*}{ Médias seguidas de mesma letra não diferem significativamente entre } \\
si pelo teste de Tukey a 5\% de significância
\end{tabular}

Tabela 1 - Análise de variância de plastocrono das diferentes datas de cultivo em Santa Maria, RS

\begin{tabular}{lccccc}
\hline Fatores de variação & Graus de liberdade & Soma de Quadrados & Quadrado Médio & Fc & Pr>Fc \\
\hline Bloco & 3 & 14,1250 & 4,7083 & 0,925 & 0,4459 \\
Datas & 7 & 47,8750 & 6,8393 & 1,344 & 0,2795 \\
Erro & 21 & 106,8750 & 5,0893 & & \\
\hline CV $(\%)$ & 9,68 & Média geral: & 23,3125 & & \\
\hline
\end{tabular}

Nível de significância a 5\% 


\section{Conclusões}

1. A temperatura base de emissão de nós é de $7,0^{\circ} \mathrm{C}$ para a melancieira, cultivar "Crimson Sweet";

2. Considerando-se as datas de cultivo estudadas o plastocrono da melancia é em média $23,3^{\circ} \mathrm{C}$ dia nó ${ }^{-1}$.

\section{Referências}

ARNOLD, C. Y. Maximum-minimum temperatures as a basis for computing heat units. Proceedings of the American Society for Horticultural Sciences, v. 76, n. 01, p. 682-692, 1960.

BASTOS, F. G. C. et al. Efeitos de espaçamentos entre plantas na cultura da melancia na Chapada do Apodi, Ceará. Revista Ciência Agronômica, v. 39, n. 02, p. 240-244, 2008.

BAKER, J. T.; REDDY, V. R. Temperature effects on phenological development and yield of muskmelon. Annals of Botany, v. 87, n. 03, p. 605-613, 2001.

BARBANO, M. T. et al. Temperatura base e acúmulo térmico no subperíodo semeadura-florescimento masculino em cultivares de milho no Estado de São Paulo. Revista Brasileira de Agrometeorologia, v. 9, n. 02, p. 261-268, 2001.

CARVALHO, L. C. C. de; BEZERRA, F. M. L.; CARVALHO, M. A. R. de Evapotranspiração e coeficientes de cultivo da melancia sem sementes. Revista Ciência Agronômica, v. 39, n. 01, p. 53-59, 2007.

CARVALHO, R. N. Cultivo de melancia para a agricultura familiar. Brasília-DF: EMBRAPA-SPI, 1999, 127 p.

EMPRESA BRASILEIRA DE PESQUISA AGROPECUÁRIA- EMBRAPA. Sistema Brasileiro de Classificação de Solos. 2 ed. Brasília-DF: Embrapa-CNPS, 2006. 306 p.

EMPRESA BRASILEIRA DE PESQUISA AGROPECUÁRIAEMBRAPA. Hortaliças em Número. Situação da Produção de Hortaliças no Brasil, 2008. Disponível em: <http://www.cnph. embrapa.br/paginas/hortalicas_em_numeros/hortalicas_em_ numeros.htm>Acesso em: 12 abril 2010.

GILMORE JUNIOR., E. C.; ROGERS, J. S. Heat units as a method of measuring maturity in corn. Agronomy Journal, v. 50, n. 10, p. 611-615, 1958.

GRAMIG, G. G.; STOLTENBERG, D.E. Leaf appearance base temperature and phyllochron for common grass and broad leaf weed species. Weed Technology, v. 21, n. 03, p. 249-254, 2007.

HELDWEIN, A. B. et al. A. O clima de Santa Maria. Ciência \& Ambiente, v. 38, p. 43-58, 2009.
HODGES, T. F. Predict crop phenology. Boca Raton: CRC, 1991. $233 \mathrm{p}$.

IBGE- Instituto Brasileiro de Geografia e Estatística. Produção Agrícola Municipal SCP/DEPLAN. 2008. Disponível em: <http://www.sidra.ibge.gov.br> Acesso em: 05 outubro 2009.

JEFFERIES, R. A.; MACKERRON, D. K. L. Thermal time as a non-destructive method of estimating tuber initiation in potatoes. Journal of Agricultural Science, v. 108, n. 01, p. 249-252, 1987.

LEÃO, D. S. S. et al. Produtividade de melancia em diferentes níveis de adubação química e orgânica. Bioscience Journal, v. 24, n. 04, p. 32-41, 2008.

LEONEL, L. A. K. et al. Produtividade de sete genótipos de melancia em Dourados. Horticultura Brasileira, v. 18, n. 03, p. 222-224, 2000.

MARTINS, F. B. et al. Estimativa da temperatura base para emissão de folhas e do filocrono em duas espécies de Eucalipto na fase de muda. Revista Árvore, v. 31, n. 02, p. 373-381, 2007.

McMASTER, G. S.; WILHELM, W. W. Growing degree-days: one equation, two interpretations. Agricultural and Forest Meteorology, v. 87, n. 02, p. 291-300, 1997.

PAULA, G. M. de; STRECK, N. A. Temperatura base para emissão de folhas e nós, filocrono e plastocrono das plantas daninhas papuã e corriola. Ciência Rural, v. 38, n. 09, p. 2457-2463, 2008.

SINCLAIR, T. R. et al. Sugarcane leaf area development under field conditions in Florida, USA. Field Crops Research, v. 88, n. 01, p. 171-178, 2004

SINCLAIR, T. R. et al. Comparison of vegetative development in soybean cultivars for low-latitude environments. Field Crops Research, v. 92, n. 01, p. 53-59, 2005.

STRECK, N. A. A. Generalized non linear air temperature response function for node appearance rate in muskmelon (Cucumis melo L.). Revista Brasileira de Agrometeorologia, v. 10, n. 01 , p. $105-111,2002$.

STRECK, N. A. et al. Incorporating a chronology response function into the prediction of leaf appearance rate in winter wheat. Annals of Botany, v. 92, n. 02, p. 181-190, 2003.

STRECK, N. A. et al. Estimativa do plastocrono em meloeiro (Cucumis melo L.) cultivado em estufa plástica em diferentes épocas do ano. Ciência Rural, v. 35, n. 02, p. 1275-1280, 2005.

STRECK, N.A. et al. Estimativa do plastocrono em cultivares de soja. Bragantia, v.67, n. 01, p. 67-73, 2008.

TRENTIN, R. et al. Soma térmica de subperíodos do desenvolvimento da planta de melancia. Ciência Rural, v. 38, n. 09, p. 2464-2470, 2008. 